\title{
Economic and Environmental Impacts on the Portfolio of Clean Energy Vehicles in Japan
}

\author{
Jun Osawa ${ }^{1}$ and Masaru Nakano ${ }^{1}$ \\ ${ }^{1}$ The Graduate School of System Design and Management, Keio University \\ Kyosei Building, 4-1-1, Hiyoshi, Kohoku-ku, Yokohama, Kanagawa, 223-8526, Japan \\ jun.osawa@keio.jp, nakano@sdm.keio.ac.jp
}

\begin{abstract}
The introduction of clean energy vehicles (CEVs) is one of the measures expected to be employed against global warming. Because the structures of CEV parts are different from those of gasoline vehicles, the popularization of each CEV type has two different types of impacts: economic and environmental. Therefore, it is necessary to consider the optimal mix of CEVs before deciding upon a policy for the introduction of CEVs. However, most conventional studies do not consider the economic effects on industries. This paper proposes a new optimization model considering the production-induced effects as well as environmental impacts. The model is applied to estimate the impacts on the portfolio of CEVs in 2030 in Japan.
\end{abstract}

Keywords: Automotive industry, Global warming, Optimization, Productioninduced effects, Sustainable design

\section{Introduction}

Japan accounts for approximately $4 \%$ of global $\mathrm{CO}_{2}$ emissions [1]. In particular, automobiles cause approximately $15 \%$ of domestic $\mathrm{CO}_{2}$ emissions [2], leading to increased calls for measures to reduce these emissions. One such measure under consideration is the introduction of clean energy vehicles (CEVs) such as clean diesel vehicles (CDVs), electric vehicles (EVs), hybrid electric vehicles (HEVs), plug-in hybrid electric vehicles (PHEVs), and fuel cell vehicles (FCVs). However, each type of CEV has its own strengths and weaknesses and differs in its energy source, fuel cost, $\mathrm{CO}_{2}$ emissions, and other elements. Thus, the widespread adoption of only one type of vehicle that may be superior in just one metric is not the best solution; rather, a portfolio comprising a mix of gasoline vehicles (GVs), diesel vehicles (DVs), natural gas vehicles (NGVs), and CEVs is needed to optimize $\mathrm{CO}_{2}$ emissions, costs, and other metrics.

Among the existing research on automobile portfolios, Ichinohe et al. [3] and Yeh et al. [4] used the MARKAL energy system model to calculate passenger vehicle portfolios that achieve the target level of $\mathrm{CO}_{2}$ emissions in Japan and the US. In addition, Yamada et al. [5] set fuel costs and vehicle purchase costs as constraints to calculate a domestic passenger vehicle portfolio that generates the minimum possible life cycle $\mathrm{CO}_{2}\left(\mathrm{LCCO}_{2}\right)$. The studies of Ichinohe et al. [3], Yeh et al. [4], and Yamada et 
al. [5] differed in their models and regions but all essentially focused on the $\mathrm{CO}_{2}$ emissions of CEVs. In contrast, in addition to $\mathrm{CO}_{2}$ emissions, Kato et al. [6] calculated the optimal portfolios of global passenger vehicles focusing on copper resource constraints. Meanwhile, Arimori et al. [7] focused on oil consumption constraints in addition to $\mathrm{CO}_{2}$ emissions in calculating the optimal portfolios of domestic passenger vehicles, trucks, and busses. In other words, Kato et al. [6] and Arimori et al. [7] expanded the definition of environmental properties from $\mathrm{CO}_{2}$ emissions alone to resource and energy sustainability.

The above examples show that prior research has primarily focused on $\mathrm{CO}_{2}$ emissions and resource and energy consumption (Figure 1, red line). In addition, the abovementioned studies did not consider economics, including changes in the production volumes of companies in the battery and automotive industries, the producers of CEVs. However, the composition of parts differs among the types of CEVs (e.g., GVs, DVs, NGVs, and others). Our previous research [8] focused on the differences between the components utilized in CEVs and GVs. By using the newly created Input-Output Table that added departments of CEVs in our research, productioninduced effects will decline by 1.5 trillion yen from 2010 to 2030 under a sales target established by the Ministry of the Environment. In particular, the automotive and auto accessories industries in Japan account for approximately $15 \%$ of manufacturing industry production. Therefore, any changes to the parts have great impacts for industry of Japan. However, in that study, we did not discuss environmental issues and automobile portfolios.

\section{Clean energy vehicles}

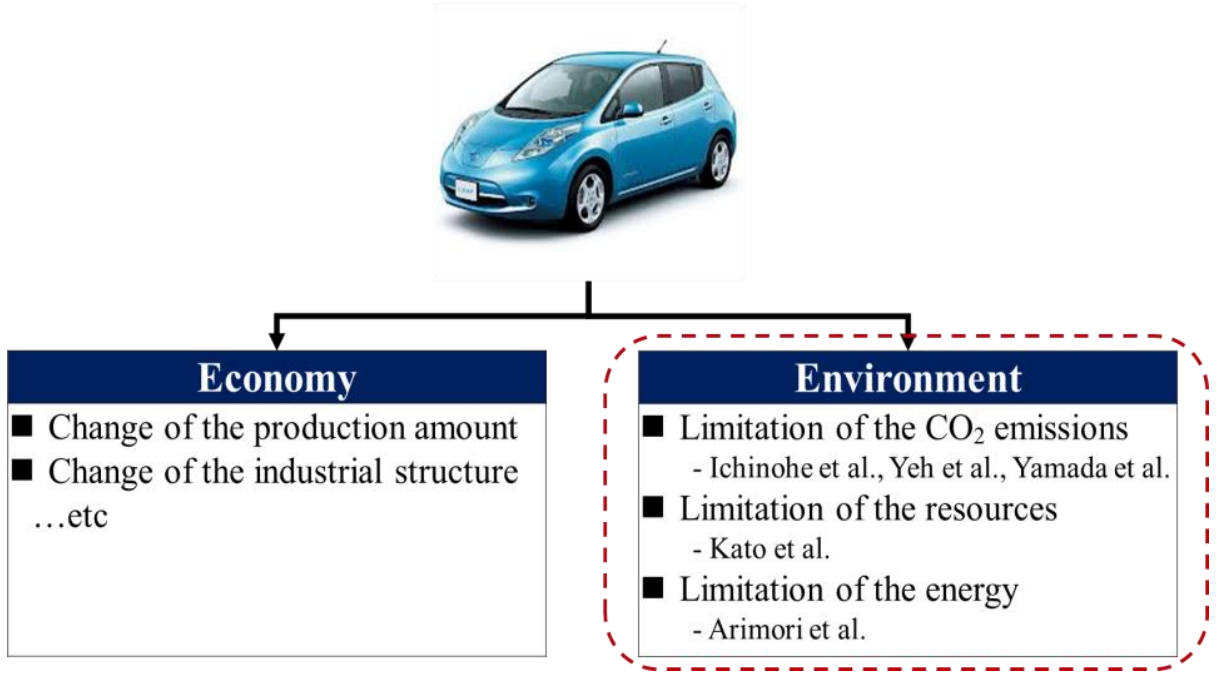

Fig. 1. Research area of portfolio optimization for clean energy vehicles

Therefore, we propose a new model that optimizes portfolios by considering both the economic and environmental impacts of CEVs. The aim of this study was to de- 
termine the optimal portfolio for domestic passenger vehicles in order to assist corporate product plans for CEVs along with governmental policies for spreading CEVs. Eight types of passenger vehicles were covered in this study: GVs, DVs, CDVs, NGVs, EVs, HEVs, PHEVs, and FCVs.

In chapter 2 , we describe a newly created optimization model. In chapter 3 , we calculate an optimal portfolio in each case using the new optimization model. In chapter 4 , we summarize our research and discuss future issues.

\section{Optimization model}

\subsection{Framework}

As noted above, in this study, we created an optimization model that considers the economics of CEVs in addition to $\mathrm{CO}_{2}$ emissions and consumer cost. Consumer cost is defined as the sum of fuel costs incurred when driving and the vehicle purchase costs. CEV economics were evaluated using production-induced effects caused by $\mathrm{CEV}$ adoption. Production-induced effects indicate the total production volumes directly and indirectly required in each industry to meet the demand generated by producing a final good. In this study, this volume would be the total production volume of each industry when each model of car is consumed. In addition, this study also considered the variance in production-induced effects for each type of CEV, and the Input-Output Table ("Input-Output Table for CEVs") created by the author [8] was used as part of the optimization model because traditional Input-Output Tables are only divided into two types of vehicles: "passenger vehicles" and "other vehicles." Thus, we could not have analyzed the variance in production-induced effects for each type of CEV by using traditional Input-Output Tables. On the basis of the differences between the components utilized in each CEV, our Input-Output Table for CEVs was expanded from two types of vehicles to a total of twenty four types: passenger vehicles, trucks, and buses along with GVs, DVs, CDVs, NGVs, EVs, HEVs, PHEVs, and FCVs in each of the first three categories. Thus, by using our Input-Output Table, we could quantify the impacts of CEV adoption on production volumes and consider the economic characteristics of CEVs in the calculation of an optimal portfolio.

Figure 2 represents the optimization model built for this study. Our model can calculate the optimal mix of CEVs on an annual basis by inputting some assumptions after deciding constraints and an objective function. The production-induced effects and $\mathrm{CO}_{2}$ emissions within the optimal solution are calculated simultaneously.

Next, we define "optimal" as it is used in this paper. We set two cases of objective functions and conducted a what-if analysis. The first case was an objective function of production-induced effects across all industries caused by the adoption of CEVs. The second case set an objective function for $\mathrm{CO}_{2}$ emissions. We then solved this optimization problem using $\mathrm{CO}_{2}$ emissions and consumer cost as constraints. "Optimization" in Case 1 of this study refers to a situation in which production-induced effects are the largest, even when $\mathrm{CO}_{2}$ emissions and consumer cost constraints are met. "Op- 
timization" in Case 2 highlights a situation in which $\mathrm{CO}_{2}$ emissions are the smallest, even when consumer cost constraints are met.

In this study, to derive an optimal solution, we created an optimization model in spreadsheet form using Microsoft Excel and used the linear program (Simplex LP) in "Solver Function."

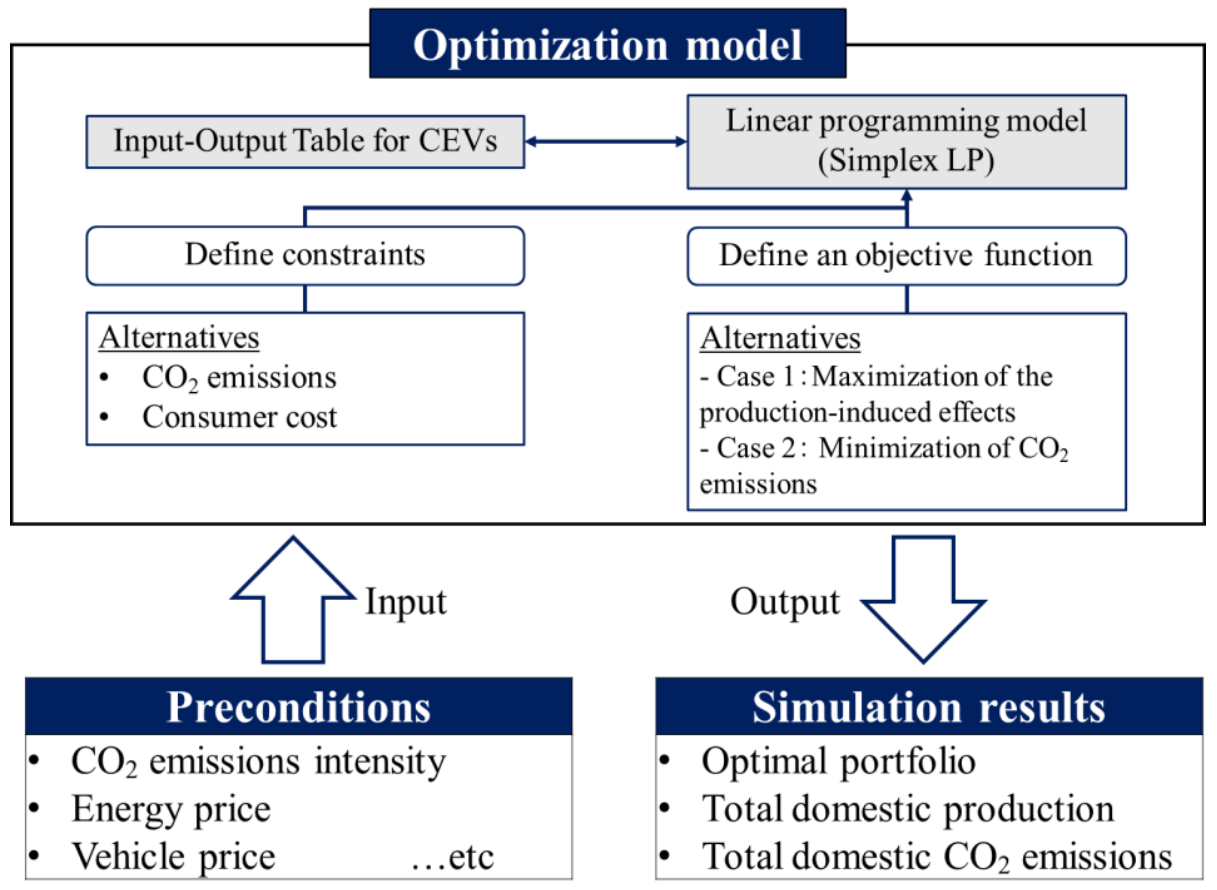

Fig. 2. Outline of CEV portfolio optimization model

\subsection{Objective function}

For Case 1, we set an objective function for the production-induced effects in Japan and formulated it as formula (1). In addition, we employed a numerical formula from a previous study [8] to calculate the production-induced effects.

The production-induced effects were calculated by multiplying the direct effects by the Leontief inverse matrix. The Leontief inverse matrix represents the "sum of direct and indirect spillovers generated in each industry by the consumption of one unit of a final good" [9]. The direct effects were defined based on three perspectives (the costs of production, fuel, and construction of the service station) for each vehicle type in a previous study [8].

$$
\max f_{k}\left(X_{i k}\right)=\{I-(I-M) A\}^{-1} D_{k}
$$


$i$ : Type of passenger vehicle $\quad[\mathrm{GV}, \mathrm{DV}, \mathrm{CDV}, \mathrm{NGV}, \mathrm{EV}, \mathrm{HEV}, \mathrm{PHEV}, \mathrm{FCV}]$

$k$ : Target year

$f_{k}$ : Production-induced effects in year $k$ [yen]

$X_{i k}$ : New sales of vehicle type $i$ in year $k$ [unit]

I: Identity matrix

$M$ : Imports matrix

$A$ : Input coefficient table

$D_{k}$ : Direct effects in year $k \quad$ [yen]

Next, we set a Case 2 objective function for the $\mathrm{CO}_{2}$ emissions in Japan and formulated it as follows:

$$
\min g_{k}\left(X_{i k}\right)=\sum_{i} X_{i k} U_{i k}
$$

$g_{k}: \mathrm{CO}_{2}$ emissions in year $k \quad\left[\mathrm{~kg}-\mathrm{CO}_{2}\right]$

$U_{i k}$ : $\mathrm{CO}_{2}$ emissions of a vehicle type $i$ in year $k \quad\left[\mathrm{~kg}-\mathrm{CO}_{2} / \mathrm{unit}\right]$

\subsection{Constraint conditions}

In this study, we set two constraints: consumer cost and $\mathrm{CO}_{2}$ emissions.

Formula (3) expresses the limitation on consumer cost for any year $k$. Consumer cost consists of fuel costs and vehicle purchase costs. Formula (4) shows the limitation on $\mathrm{CO}_{2}$ emissions for any year $k$.

$$
\begin{gathered}
\sum_{i} X_{i k^{0}}\left(P_{i k^{0}}+E_{i k^{0}}\right) \geq \sum_{i} X_{i k}\left(P_{i k}+E_{i k}\right) \\
\sum_{i} T S_{i k} U_{i k} \geq g_{k}\left(X_{i k}\right)
\end{gathered}
$$

$k^{0}$ : Base year [2010]

$P_{i k}$ : Sales price of a vehicle type $i$ in year $k$ [yen/unit] $E_{i k}$ : Fuel costs of a vehicle type $i$ in year $k$ [yen/unit]

$T S_{i k}$ : Target unit sales of vehicle type $i$ in year $k$ according to the Ministry of the Environment [unit]

\section{Simulation results}

In this chapter, we calculate the optimal portfolio of the passenger vehicles in 2030 in each case and analyze the differences. Figure 3 shows the share of the unit sales of 
passenger vehicles in 2030 for each case. The left-most bar of Figure 3 shows the target sales of the Ministry of the Environment [10] as a reference.

At first, when we maximize the production-induced effects (Case 1), HEVs and PHEVs account for approximately $90 \%$ of all components. When we minimize $\mathrm{CO}_{2}$ emissions (Case 2), HEVs play an important role, as in Case 1 (Figure 3). Conversely, EVs account for more than $40 \%$ of all components in Case 2.

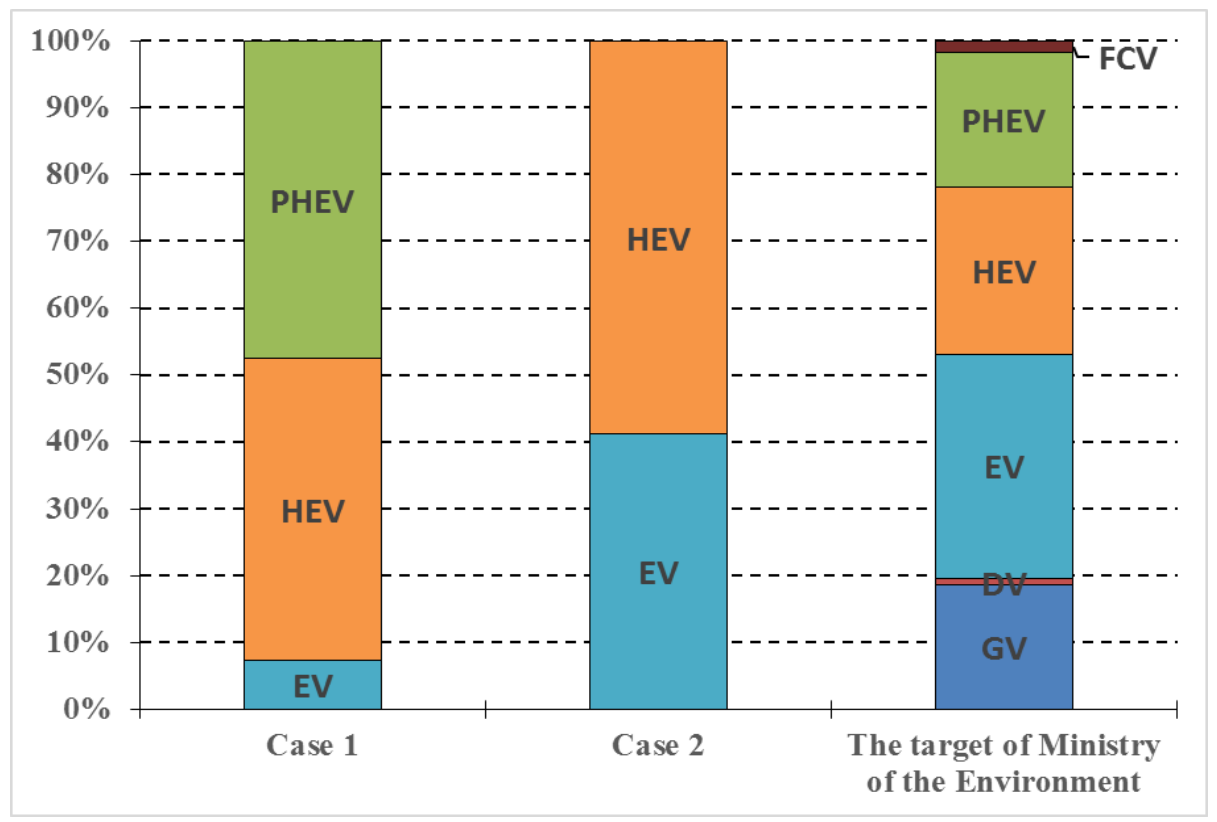

Fig. 3. Share of new sales of passenger vehicles in 2030

We think that there are two reasons for the difference between the diffusion rates of EVs and PHEVs in each case. First, EVs have superior fuel consumption and lower $\mathrm{CO}_{2}$ emissions. Therefore, the role of EVs is important when the influence on the environment is prioritized. Second, the part structure differs between EVs and PHEVs. The basic structure of a PHEV is the same as that of a GV. In addition, a battery and motor are necessary for PHEVs. Therefore, the spread of PHEVs has few negative effects on the existing auto parts industry. PHEVs also produce demand for other related industries, including the battery industry. Thus, PHEVs play an important role when we consider the influence on the economy. Although EVs have very high environmental performance, the production-induced effects of EVs are not large because some of the existing components of GVs are rendered obsolete. As a result, PHEVs are expected to become widespread in Case 1.

The above-mentioned results suggest the following conclusions. The portfolio generated with a focus on economic impacts is significantly different from that generated with a focus on the environment. These results demonstrate the importance of analyz- 
ing the portfolio of CEVs not only from an environmental perspective but also from an economic one. Also, the results indicate that the introduction of PHEVs as passenger vehicles becomes important when the influence on the economy is considered.

\section{Conclusions}

In this study, we built a new optimization model for composition of vehicle types that includes economic impacts (production-induced effects). We also calculated the optimal portfolio of passenger vehicles in Japan using the new model and analyzed its effectiveness.

As a result, the following conclusions were reached:

- When minimizing $\mathrm{CO}_{2}$ emissions is prioritized without considering economic impacts, the introduction of EVs as passenger vehicles becomes important.

- In contrast, when production-induced effects in Japan are prioritized, the spread of PHEVs as passenger vehicles becomes more important. In this case, the introduction of EVs is not very important.

- The different results for the two cases are mainly attributed to the difference between the part structures of EVs and PHEVs.

This newly created optimization model that includes economic effects will aid the industry in the adoption of CEVs.

Future works will improve the model constructed in this study and help design policies to transition CEVs into widespread use.

\section{Acknowledgments}

This work was supported in part by a Grant-in-Aid for Scientific Research from the Ministry of Education, Culture, Sport, and Technology in Japan.

\section{$5 \quad$ References}

1. IEA, $\mathrm{CO}_{2}$ emissions from fuel combustion highlights 2014, http://www.iea.org/publications/freepublications/publication/co2-emissionsfrom-fuel-combustion-highlights-2014.html (2014)

2. Ministry of the Environment, Greenhouse gas emissions in 2012, http://www.env.go.jp/earth/ondanka/ghg/index.html (2014)

3. Ichinohe, M. and Endo, E.: Analysis of the vehicle mix in the automobile sector in Japan for $\mathrm{CO}_{2}$ emission reduction by using MARKAL model. Energy and Resources, Vol. 26, No. 2, pp. 127-133 (2005)

4. Yeh, S., Farrell, A., Plevin, R., Sanstad, A. and Weyant, J.: Optimizing U.S. mitigation strategies for the light-duty transportation sector: what we learn from 
a bottom-up model. Environmental Science \& Technology, Vol. 42, No. 22, pp. 8202-8210 (2008)

5. Yamada, S. and Hondo, H.: Optimal product-replacement model considering lifetime distribution: analysis of introduction of green cars for carbon dioxide emission reduction. Energy and Resources, Vol. 30, No. 1, pp. 9-15 (2009)

6. Kato, Y., Nakano, M. and Nonaka, T.: Optimization model for global portfolio of clean energy vehicles considering metal resource. Transactions of the Japan Society of Mechanical Engineers, Series C, Vol. 79, No. 797, pp. 77-89 (2013)

7. Arimori, Y. and Nakano, M.: Portfolio optimization for clean energy vehicles in Japan. Transactions of the Japan Society of Mechanical Engineers, Series C, Vol. 78, No. 791, pp. 2571-2582 (2012)

8. Osawa, J. and Nakano, M.: A model of the economic ripple effect caused by the spread of clean energy vehicles. Transactions of the Japan Society of Mechanical Engineers, Vol. 81, No. 823, pp. 1-14 (2015)

9. Ministry of Economy, Trade and Industry, White Paper on International Economy and Trade 2012, http://www.meti.go.jp/english/report/data/gWT2012fe.html (2012)

10. Ministry of the Environment, Strategy for the spread of environmental friendly vehicles, http://www.env.go.jp/air/report/h22-02/index.html (2010) 